Sneath, P. H. A. (1955). J. gen. Microbiol. 13, 561-̃̃68

\title{
Proof of the Spontaneity of a Mutation to Penicillinase Production in Bacillus cereus
}

\author{
By P. H. A. SNEATH \\ National Institute for Medical Research, London, N.W. 7
}

\begin{abstract}
SUMMARY: A stable mutation in a strain of Bacillus cereus from penicillin sensitivity to penicillin resistance due to the production of large amounts of penicillinase is reported. By the velvet pad replicate technique this mutation has been shown to be a spontaneous event occurring in the absence of penicillin. Penicillin-resistant mutants were obtained and the penicillinase production proved, without ever bringing the cells into contact with the antibiotic. The mutation rate is about $3 \times 10^{--8}$ mutations per bacterium per division.
\end{abstract}

There is considerable argument over the respective importance of spontaneous mutation and specifically directed mutation or 'permanent adaptation' in bacteria. The example here described is important because it has been conclusively shown to be spontaneous, the mutation can be detected without treating the cells with the substrate, and the sensitivity of assay is great enough to detect a few molecules of enzyme/cell.

Most strains of Bacillus cereus produce penicillinase adaptively after pretreatment with penicillin for a few hours. Only one strain, out of 57 strains tested, failed to produce detectable enzyme even after such pretreatment; this was strain 5, which was isolated from soil (Knight \& Proom, 1950; strain CN 2501). It is a typical strain of $B$. cereus differing from the majority of strains in not fermenting salicin, and in giving a delayed digestion of starch. Upon growth overnight in nutrient broth containing 1 unit penicillin $/ \mathrm{ml}$. the culture was found to have a high content of penicillinase; on plating out the culture most of the colonies were found to produce the enzyme in large amounts without pretreatment with penicillin. One colony was purified by plating and is the mutant $5 / B$.

\section{METHODS}

The organism was routinely grown upon tryptic meat digest broth, solidified when required by $1.5 \%$ Japanese agar and incubated at $30^{\circ}$. Spore suspensions were prepared by growth on agar slopes with the addition of $1 \mu \mathrm{g}$. manganous chloride/ml.; after a week the organisms were removed, washed three times with distilled water, sealed in ampoules, and after heating at $60^{\circ}$ for 30 min., stored at $4^{\circ}$.

Tests for penicillinase activity. 'To the nutrient agar was added $4 \%$ of Andrade's indicator, and the addition of strong penicillin solution after growth of colonies gave red halos around penicillinase-producing colonies-the method described by Manson, Pollock \& Tridgell (1954). A more sensitive test is to use agar containing $1 \%$ of starch; after growth the plates are flooded with $1 \%$ 
iodine in $2 \%$ aqueous potassium iodide, the solution poured off, and strong penicillin solution $(100,000$ units $/ \mathrm{ml}$.) added. Colonies which produced penicillinase give pale halos. For strains which, unlike strain 5, digest starch rapidly this test is unsuitable, but colonies in agar without starch show pale halos on a brown background by this method.

For rough estimations of penicillinase in liquid cultures the quick assay of Perret (1954) was used with a calibration curve.

Penicillin. Crystalline sodium benzylpenicillin from Glaxo Laboratories Ltd., Greenford, Middlesex was used. Penicillin plates were made by adding freshly made solutions of the antibiotic to melted agar at $56^{\circ}$, and poured within a few minutes.

Plating technique. To avoid spreading of the organism in surface colonies and between the agar and the glass, sandwich layer plates were used, the bacteria being incorporated into the middle layer of medium. All three layers were of c. $15 \mathrm{ml}$. of tryptic meat nutrient agar with indicator.

Estimation of the proportion of mutants in a culture. The number of mutants in a culture could be estimated with reasonable accuracy, as determined by reconstruction experiments, by sandwich pour plates containing one of the penicillinase indicators, and 1 unit penicillin/ml., provided the total inoculum was not more than about $5 \times 10^{7}$ cells. Rather heavier inocula could be used when 2 units penicillin/ml. were added. At these concentrations of penicillin almost all the mutants form colonies and the parent cells fail to grow. Incubation was for $18 \mathrm{hr}$. at $30^{\circ}$. Only penicillinase-positive colonies were scored as mutants.

Velvet pad replicate technique. The technique devised by Lederberg \& Lederberg (1952) was used with modifications as follows. Commercial velveteen with a short nap was cut into convenient squares, washed in distilled water and sterilized by autoclaving. A square was placed over the end of a waxed wooden cylinder $8 \mathrm{~cm}$. in diameter, and held in place by an elastic band. Two sterile pins were placed in the velvet as markers of position. The block was soaked in hypochlorite solution (Chloros) and then rinsed in sterile water and dried with sterile absorbent cotton-wool before use.

Single spores of strain 5 were plated on nutrient agar. From this plate a single colony was spread on a nutrient agar 'master plate' to give a confluent growth, and after about $6 \mathrm{hr}$. of incubation this master plate was pressed on sterile velveteen (with pins for orientation), and was set aside at $4^{\circ}$ until the next day. A number of penicillin agar plates ( 2 units $/ \mathrm{ml}$.) were then pressed in succession on the velveteen, and well dried. Since surface spreading of the growth was troublesome on the replica plates, it was necessary to touch in the imprints of the pins with sterile indian ink, to mark their position, and then to superimpose a layer of penicillin agar. This layer does not appear to disturb the position of the cells. These replica plates were incubated overnight. When resistant colonies were found at the same spots (relative to the pin marks) upon two or more of the plates, it was deduced that they were due to cells from a resistant micro-colony upon the master plate in the corresponding position. Two or three such areas of the master plate presumed to contain resistant 
clones (which must have arisen spontaneously since they had not been in contact with penicillin at any time) were cut out, and emulsified in broth. This broth was used to inoculate another master plate and the cycle repeated until a pure culture of mutants was obtained. The velveteen was used again after soaking in dilute Chloros (1/10) for 5 min., washing well in tap water and autoclaving.

\section{RESULTS}

\section{Characteristics of the parent strain and the penicillinase-producing} mutant strains

The parent and the mutant $\mathbf{5} / \mathbf{B}$ have the same colony form and biochemical behaviour apart from penicillinase production. No gross difference in growth rate was detected by inoculating equal numbers of viable spores of 5 and $5 / \mathrm{B}$ into the same tube of broth, and subculturing the mixed culture daily three times: the last broth culture still contained $50 \%$ of mutants (within the limits of the counting error). The parent strain contains no penicillinase detectable by the quick assay of Perret (1954) or the manometric method of Henry \& Housewright (1947), and is unable to produce it inductively; the mutant produces large amounts of penicillinase constitutively, and there is no appreciable increase when treated with penicillin. The sensitivity to penicillin is shown in Table 1. It is seen that single spores of the mutant are resistant to 4 units penicillin $/ \mathrm{ml}$., while spores of the parent are inhibited by $0.03 \mathrm{unit} / \mathrm{ml}$.

Mutant 5/B has not lost its penicillinase-producing ability after many subcultures in penicillin-free media, and so far no penicillinase-negative colonies have been detected in subcultures. The mutant produces penicillinase, which has been crystallized, in an amount equivalent to $2 \%$ of the dry weight of the cells (Pollock, Torriani \& Tridgell, 1956). Penicillin-resistant variants which did not produce penicillinase were not found.

Table 1. Penicillin sensitivity of Bacillus cereus strains 5 and $5 / B$ when samples of about* 50 spores were plated in penicillin nutrient agar

Penicillin concentration (units/ml.)

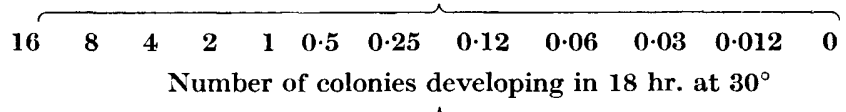

Parent (strain 5)

Mutant (strain 5/B)

$\begin{array}{rrrrrrrrrrrr}0 & 0 & 0 & 0 & 0 & 0 & 0 & 0 & 0 & 0 & 59 & 51 \\ 0 & 20 & 42 & 57 & 50 & 50 & 64 & 72 & 55 & 59 & 61 & 66\end{array}$

* About 50 spores since actual number varied according to the usual random error.

\section{Selection of mutants by growth in penicillin media}

It was suspected that the enzyme-producing variants were of mutational origin when it was observed that plating of about $10^{7}$ spores of the parent in penicillin plates ( 1 or 2 units $/ \mathrm{ml}$.) gave a constant number of resistant penicillinase-producing colonies, surrounded by satellite colonies of the parent. The latter evidently arose from organisms of the parent type which had 
survived until the variant colony had destroyed the penicillin in the immediate vicinity. Other parts of the plate were sterile or almost so after $24 \mathrm{hr}$. of incubation. When single spore clones of the parent strain were prepared, they also all gave mutants when plated in this fashion using inocula of $10^{7}$ organisms/ plate.

In penicillin broth ( 1 unit $/ \mathrm{ml}$.) it was observed that no growth occurred with small inocula of strain 5 , while with larger inocula $\left(c .10^{6}\right.$ organisms) the culture which developed consisted almost entirely of mutants. With very large inocula the proportion of mutants after incubation was less, probably because the mutants destroyed the penicillin before all the parent organisms had been killed. There was considerable lysis of strain 5 in penicillin broth.

\section{Experiments to demonstrate the occurrence of the mutation in the absence of penicillin}

A number of techniques have been devised to decide whether mutations are induced specifically by the substance which causes selection of the mutants or whether they arise spontaneously, i.e. in the absence of the substance (Luria \& Delbrück, 1943; Newcombe, 1949; Lederberg \& Lederberg, 1952). As a preliminary test, the fluctuation test of Luria \& Delbrück (1943) was used. One of the experiments is summarized in Table 2 . It can be seen that, as required on the theory of spontaneous mutation, the variation between individual culture tubes was much greater than the expected counting errors.

\section{Table 2. Fluctuation test upon penicillin-resistant mutants of Bacillus cereus}

Twelve tubes containing $2 \mathrm{ml}$. samples of nutrient broth containing 30 viable spores $/ \mathrm{ml}$. of strain 5 , were incubated for $18 \mathrm{hr}$. at $30^{\circ}$. The number of mutants in $0.1 \mathrm{ml}$. amounts was estimated by plating in penicillin agar $(1 \mathrm{unit} / \mathrm{ml}$.). Six replicate samples were plated from tubes 1 and 12; only one sample from each of the other tubes was plated.

\begin{tabular}{|c|c|c|}
\hline Tube no. & $\begin{array}{l}\text { Mutants in } 0.1 \mathrm{ml} . \\
\text { of broth }\end{array}$ & $\begin{array}{c}\text { Total viable } \\
\text { count } / \mathrm{ml} \text {. }\end{array}$ \\
\hline 1 & $\begin{array}{c}2 \text { (replicate } \\
\text { counts =0, } 2,1 \\
2,0,1)\end{array}$ & $9 \times 10^{2}$ \\
\hline 2 & 43 & . \\
\hline 3 & 37 & . \\
\hline 4 & 6 & . \\
\hline 5 & 0 & . \\
\hline 6 & 3 & . \\
\hline 7 & 1 & . \\
\hline 8 & 4 & . \\
\hline 9 & 1 & . \\
\hline 10 & 0 & . \\
\hline 11 & 1 & . \\
\hline 12 & $\begin{array}{c}1 \text { (replicate } \\
\text { counts }=1,2,1 \\
3,2,0)\end{array}$ & $8 \times 10^{7}$ \\
\hline
\end{tabular}

The approximate mutation rate can be estimated from the data. Using the second method of Lea \& Coulson (1949) the median number of mutants/tube 
is 25 , and $m$, the mean number of mutations/tube is $\mathbf{7 \cdot 6}$. The mutation rate/ bacterium/generation is $0.69 \mathrm{~m} / \mathrm{final}$ population; in this experiment it was $3 \cdot 1 \times 10^{-8}$. Two further fluctuation tests gave estimates of $5 \cdot 6 \times 10^{-8}$ and $1 \cdot 8 \times$ $10^{-8}$. One may conclude that the mutation rate to penicillinase production is about $3 \times 10^{-8} /$ bacterium/generation.

The constant proportion of mutants, about one in $2 \times 10^{5}$, in subcultures of strain 5 (provided the cells have reached a large number such as $10^{11}$ ), demands explanation. The commonest reason given is that the mutation rate is balanced by a back-mutation rate, so that at equilibrium:

$$
\begin{aligned}
& \text { Forward mutation rate } \times \text { proportion of non-mutants } \\
& =\text { Backward mutation rate } \times \text { proportion of mutants }
\end{aligned}
$$

In this case the formula would yield the figure of $6 \times 10^{-3}$ for the back mutation rate, which is unusually high and which should have been easily detected by plating out of subcultures of mutants. No such back-mutants were detected. The more probable explanation is that the mutant cells have a slightly slower rate of growth upon the average than the parent cells. A small difference in growth rate will account for the equilibrium even in the absence of back mutation (Armitage, 1952). The relevant formula, in the absence of back mutation, is

Proportional difference in growth rates $=\lambda /$ proportion of mutants at equilibrium.

This is derived from Armitage (1952) as follows. The proportion of mutants at equilibrium $=g /(a+g-b)$ (from section 1.3); this is $(a+g) \lambda /(a+g-b)$, where $a+g$ is the growth rate of the non-mutant in Armitage's notation, and $b$ the growth rate of the mutant (section $1 \cdot 1)$. The proportional difference in growth rates $(a+g-b) /(a+g)$, equals $(g /(a+g)) \times 1 /$ proportion of mutants at equilibrium. The quantity $g /(a+g)$ is $\lambda$ given above, and is the mutation rate expressed in equation 21 (Armitage, 1952), which is the mutation rate per bacterium per division cycle (as calculated above from the fluctuation tests) divided by $0 \cdot 69$ (see equation 20, Armitage, 1952). In the instance studied here, the mutants would have a difference in average growth rate of $6 \times 10^{-3}$, i.e. a growth rate $99.4 \%$ of that of the parent, which might not be detected in a short-term experiment.

Atwood, Schneider \& Ryan (1951) also suggested that a major reason for the apparent stability of cultures of mutants and non-mutants is a constant mutation of the organisms in the majority to a form with a slightly higher growth rate, thus placing all the previous mutant organisms at a relative disadvantage, i.e. they are outgrown.

A number of objections have been made to the fluctuation test as a proof of spontaneous mutation; the most cogent is that of Dean \& Hinshelwood (1952). They point out that where a culture of organisms which can adapt to a toxic substance is used, a very slight difference in the culture tubes or the plates used for counting mutants can give great fluctuation, since the number of colonies formed is only a reflexion of the number of cells which are able to 
become adapted before they are killed. This may depend on many uncontrollable factors. I have confirmed this in Bacillus cereus strain $\mathbf{5 6 9}$, which produces penicillinase adaptively; the number of colonies formed on penicillin plates fluctuates widely at certain critical levels of penicillin. It is therefore preferable to use another test for proof of spontaneity of mutations.

\section{Use of the velvet pad replicate technique}

This technique devised by Lederberg \& Lederberg (1952) is the only one which gives unambiguous proof of the spontaneity of a mutation, since by this method one can isolate a mutant without ever exposing it to the selecting agent concerned. The test was performed successfully on three occasions, yielding each time a penicillinase-producing strain. On one occasion a distinctive colonial variant of strain 5 was used, which yielded a mutant of the same colonial type, thus excluding the possibility of chance contamination of the plates by a penicillinase-producing bacterium. The number of 'cycles' needed for isolation of a mutant was five or six. The degree of enrichment at each step is indicated by the percentage of mutants present in the broth used to inoculate the successive master plates in one series: 1st master plate, $0.00002 \%$; 2nd, $0.0004 \%$; 3rd, $0.01 \%$; 4th, $0.07 \%$; 5th, $0.1 \%$; 6th, $72 \%$; final culture, $100 \%$.

\section{DISCUSSION}

The velvet-pad technique, though published in 1952, has been little used for proving that mutations are spontaneous. There seem to be only four published examples of its use for this purpose: Lederberg \& Lederberg (1952) showed that mutations to resistance to streptomycin and phage $\mathrm{T}_{2}$ in Escherichia coli were spontaneous; Kraft \& Braun (1955) found mutations causing antibiotic resistance to be spontaneous in Brucella suis and Escherichia coli; Goldstein (1954) showed that streptomycin resistant and dependent mutations occurred spontaneously in E. coli; Lam \& Sevag (1955) used the method for studying the origin of streptomycin resistance. It is uncertain whether the latter authors fully understood the principles of this test; as they apparently only carried out a single 'cycle', it is most unlikely that the degree of enrichment would have given a population consisting mainly of resistant cells. Their failure to obtain resistant clones is therefore not unexpected.

There are only two formal objections to the technique: (1) that traces of antibiotic remain on the velveteen if it is used repeatedly, or in some other way the media become contaminated by traces of antibiotic; (2) the 'resistant' culture consists not of resistant organisms, but of organisms which are able to become resistant with great facility when they come into contact with the antibiotic. The latter behaviour could of course be due to selection either of a spontaneously arising variant with an exceedingly high rate of mutation which is specifically induced by the antibiotic, or of one composed of organisms which can all adapt to resistance by a mechanism similar to enzyme induction (for most antibiotics only a theoretical possibility). In the instance here 
described, the first objection is answered by the great lability of aqueous penicillin to heat, so that there were probably no traces in the velveteen or the media. The second objection is answered by the observation that Seitz filtrates of penicillin-free broth cultures of the three mutant clones contained large amounts of penicillinase; in view of the close correlation found between resistance and penicillinase production, it would seem reasonable to attribute the resistance to the presence of the enzyme. Therefore the agent responsible for resistance was produced in the absence of penicillin. It is not possible with most systems to demonstrate this since the organisms have to be treated with the antibiotic in order to prove that they are resistant.

It should be noted that absence of a positive result does not disprove spontaneity - it may be due, for instance, to slow growth of the mutant which nullifies the enrichment obtained in each step. At present, also, there is no way of estimating the number of mutations/plate because of the uncertainty of the amount of organisms transferred by the velveteen pad. The test is, however, so decisive when it is positive that it is well worth using despite its laboriousness. Once a change is proved to occur to some extent spontaneously, to prove specifically-directed mutation one must show that the antibiotic increases the rate of mutation, a problem beset with many technical difficulties as is well-known, but only too frequently ignored.

It should be noted that although the Bacillus cereus strain $\mathbf{5}$ is described here as penicillinase-negative, there is some evidence for a larger amount of enzyme in the cultures than is accounted for by the number of penicillinaseproducing mutants present (Pollock \& Sneath, to be published). This is being further investigated; the penicillinase content of the parent organisms must, however, be very small, of the order of one molecule/organism.

\section{REFERENCES}

Armitage, P. (1952). The statistical theory of bacterial populations subject to mutation. J. R. statist. Soc. B, 14, 1.

Atwood, K. C., Schneider, L. K. \& Ryan, F. J. (1951). Periodic selection in Escherichia coli. Proc. nat. Acad. Sci., Wash. 37, 146.

Dean, A. C. R. \& Hinshelwood, C. (1952). The resistance of Bact. lactis aerogenes to proflavine (2:8-diaminoacridine). I. The applicability of the statistical fluctuation test. Proc. Roy. Soc. B, 139, 236.

Gol.Dstein, A. (1954). The origin of streptomycin-dependent variants of Escherichia coli. J. Pharmacol. 112, 326.

Henry, R. J. \& Housewright, R. D. (1947). Studies on penicillinase. II. Manometric method of assaying penicillinase and penicillin, kinetics of the penicillinpenicillinase reaction, and the effects of inhibitors on penicillinase. J. biol. Chem. $167,559$.

Knight, B. C. J. G. \& Proom, H. (1950). A comparative survey of the nutrition and physiology of mesophilic species in the genus Bacillus. J.gen. Microbiol. 4, 508.

Kraft, M. \& Braun, W. (1955). Observations on replica plating and antibioticresistance. Bact. Proc. 52.

Lam, G. T. \& Sevag, M. G. (1955). Mechanism of the development of resistance to streptomycin. II. Biochemical differences of replicate colonies. J. Bact. 69, 184.

LEA, D. E. \& Coulson, C. A. (1949). The distribution of the numbers of mutants in bacterial populations. J. Genet. 49, 264. 
Lederberg, J. \& Lederberg, E. M. (1952). Replica plating and indirect selection of bacterial mutants. J. Bact. 63, 399.

Luria, S. E. \& Delbrück, M. (1943). Mutations of bacteria from virus sensitivity to virus resistance. Genetics, 28, 491.

Manson, E. E. D., Pollock, M. R. \& Tridgeli, E. J. (1954). A comparison of the properties of penicillinase produced by Bacillus subtilis and Bacillus cereus with and without addition of penicillin. J. gen. Microbiol. 11, 493.

Newcombe, H. B. (1949). Origin of bacterial variants. Nature, Lond. 164, 150.

Perret, C. J. (1954). Iodometric assay of penicillinase. Nature, Lond. 174, 1012.

Pollock, M. R., Torriani, A.-M. \& Tridgell, E. J. (1956). Crystalline bacterial penicillinase. Biochem. $J$. (in the Press).

(Received 8 July 1955) 